\title{
Optical and A.C. Electrical Properties for Polypyrrole and Polypyrrole/Graphene (ppy/gn) Nanocomposites
}

\author{
Fatima Mikdad Ahmed ${ }^{1 \mathrm{a} *}$ and Salma Mohammad Hassan ${ }^{1 \mathrm{~b}}$ \\ ${ }^{1}$ Department of Physics, College of Science, University of Baghdad, Baghdad, Iraq \\ bE-mail: salma.mhammed@yahoo.com \\ a*Corresponding author: alameenmoqdad@gmail.com
}

\begin{abstract}
In this work, chemical oxidation was used to polymerize conjugated polymer "Polypyrrole" at room temperature Graphene nanoparticles were added by in situ-polymerization to get (PPY-GN) nano. Optical and Electrical properties were studied for the nanocomposites. optical properties of the nanocomposites were studied by UV-Vis spectroscopy at wavelength range $(200-800 \mathrm{~nm})$. The result showed optical absorption spectra were normally determined and the result showed that the maximum absorbance wave length at $280 \mathrm{~nm}$ and $590 \mathrm{~nm}$. The optical energy gap has been evaluated by direct transition and the value has decreased from (2.1 eV for pure PPy) to (1.3 eV for 5 $\%$ wt. of GN). The optical constants such as the band tail width $\Delta \mathrm{E}$ was evaluated, the value of $\Delta \mathrm{E}$ for pure PPy was $(0.0949 \mathrm{eV})$ while for $5 \mathrm{wt}$. $\%$ of $\mathrm{GN}$ it was $(0.5156 \mathrm{eV})$, It has been observed that the Urbach tail for pure PPy was smaller than that for PPy/GN nanocomposites and it increase as GN concentration increases. The A.C electrical conductivity at range of frequency $\left(10^{3} \mathrm{~Hz}-10^{6} \mathrm{~Hz}\right)$ was increased by increasing the frequency and GN concentration about four order of magnitude. The $\mathrm{s}$ value was about (0.653-0.962) which means that the mechanism of conductivity is correlated hopping mechanism (C. H. P.). The dielectric constant and dielectric lose were determined and found to decrease with increasing frequency.
\end{abstract}

Article Info.

Keywords:

Optical properties, polypyrrole, graphene, A.C, in situ polymerization

\section{Article history:}

Received: Jan. 12, 2021

Accepted: Nov. 05, 2021

Published: Dec. 01,2021

\section{Introduction}

Polypyrrole (PPy) is a chemical material consisted of a number of joined pyrrole rings [1]. Polypyrrole (PPy) is a nondegenerate ground state polymer with two types of chain arrangement including repeating units of aromatic as well as quinoid [2], as shown in Figure (1). Pure polypyrrole (PPy) with benzenoid structure is an insulator in nature, so it needs to be oxidant by doping to act as a semiconductor [3].

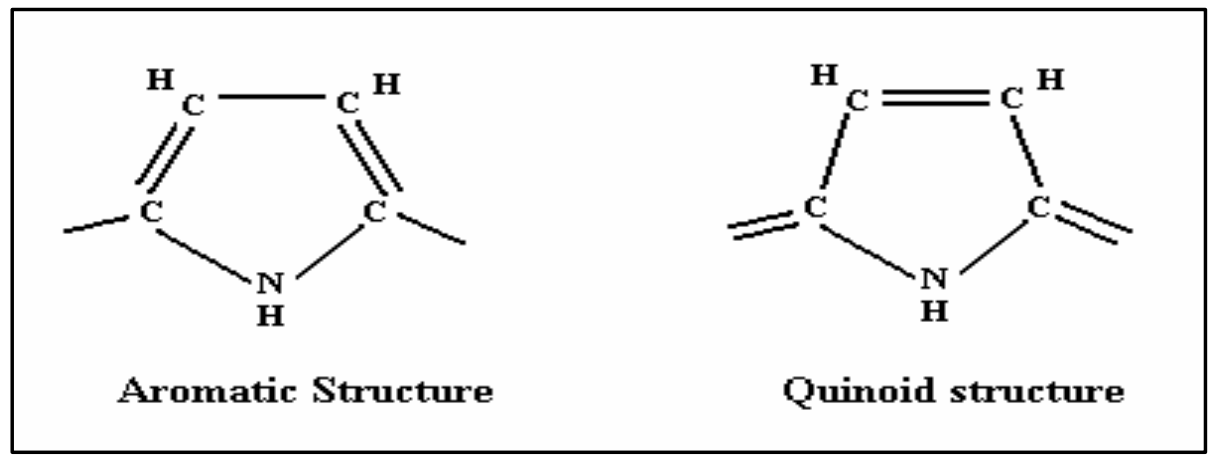

Figure 1: The Structures of polypyrrole (Aromatic and Quinoid) [3]. 
Polypyrrole (PPy) is most attractive for its simple polymerization and many application in optical switching divides, anti-static films, light emitting diodes, electromagnetic shielding layer, electro-chromic devices, electrode for batteries, photovoltaic, and gas sensors[4, 5], moreover Polypyrrole (PPY) is used in the design of solid-state electronic devices such as metal-insulator-semiconductor FET structures and metal-semiconductor because of the availability of a wide range of energy band gaps[6].

A feature of a conductive polymer is the presence of conjugated double bands across the polymer backbone. In conjugation, the bands between the carbon atoms are alternate. Single double and single. Every band has a localized sigma $(\sigma)$ band which composes a strong chemical band, and every double band have delocalized pi $(\pi)$ band which is a lower strength chemical band. However, conjugation is not enough, to make the polymer material conductive. Furthermore, to the presence of the double bonds, doping adds charge carriers in the shape of extra electrons or holes into the polymer matrix which results in increasing its electrical conductivity [7, 8].

\section{Experimental work}

Pyrrole monomer(mPy) with purity $98 \%$ was supplied by Sigma Aldrich company. Ferric chloride ( $\mathrm{FeCl} 3$ ) with purity $98 \%$, was supplied by Himedia Laboratories Pvt. Ltd, which was used as the oxidizing agent. Graphene (GN) with purity $98 \%$ and with a diameter of (6-8) nm was supplied by USA-diameter (6-8) nm Dimethely sulfoxide (DMSO) with purity $99.6 \%$ was supplied by BDH-England.

\subsection{Synthesis of pure Polypyrrole (PPy) and PPy/GN}

Polypyrrole (PPy)and PPy/GN were produced by chemical oxidative in-situ polymerization technique the use of pyrrole monomer and ferric chloride $\left(\mathrm{FeCl}_{3}\right)$ as oxidant. Pure polypyrrole was prepared by adding $(2 \mathrm{~mL})$ of pyrrole to $(150 \mathrm{ml})$ of distilled water in a beaker and stirred for (10 minutes). $(3 \mathrm{~g})$ of ferric chloride was added slowly to the pyrrole solution and stirred for (4 -5 hours) at room temperature $\left(23^{\circ} \mathrm{C}\right)$. The polypyrrole precipitate was obtained by filtration, washed several times with distilled water and dried in an oven at $70{ }^{\circ} \mathrm{C}$ for six hours. In the same way, the PPy/GN nanocomposites were prepared, but different weights $(0.1,0.3,0.5,1,3$ and 5 wt. \%) of graphene nanoparticles were added during polymerization.

\subsection{Sample Preparation for UV-Vis. Measurements}

Polypyrrole and PPy/GN nano-composites of $0.05 \mathrm{~g}$ weight were dissolved in $5 \mathrm{ml}$ DMSO. The solution was stirred with a magnetic stirrer for more than two hours to achieve a homogenous solution. A Shimadzu UV-Vis. spectrophotometer in the wavelength range $200-800 \mathrm{~nm}$ was used to is a term that is used to describe the absorption of a substance. the absorption spectra of the prepared samples. The optical energy gap was estimated using the absorption spectrum.

\subsection{Sample Preparation for Electrical properties}

$\mathrm{AC}$ electrical measurements were employed to investigate pure PPy and PPy/GN nanocomposites. The polypyrrole was thoroughly grounded in a mortar to obtain very fine particles, and then it was compressed under a pressure of ( 8 tones) to get the samples as pellets. The resulting pellet has a diameter of $(1.3 \mathrm{~cm})$ and a thickness of $(0.112$ $0.137 \mathrm{~cm}$ ), as shown in Figure (2). The sample was placed in a holder specially designed to minimize lost capacitance and leakage current (guard ring electrode methods), as shown in Figure (3). 


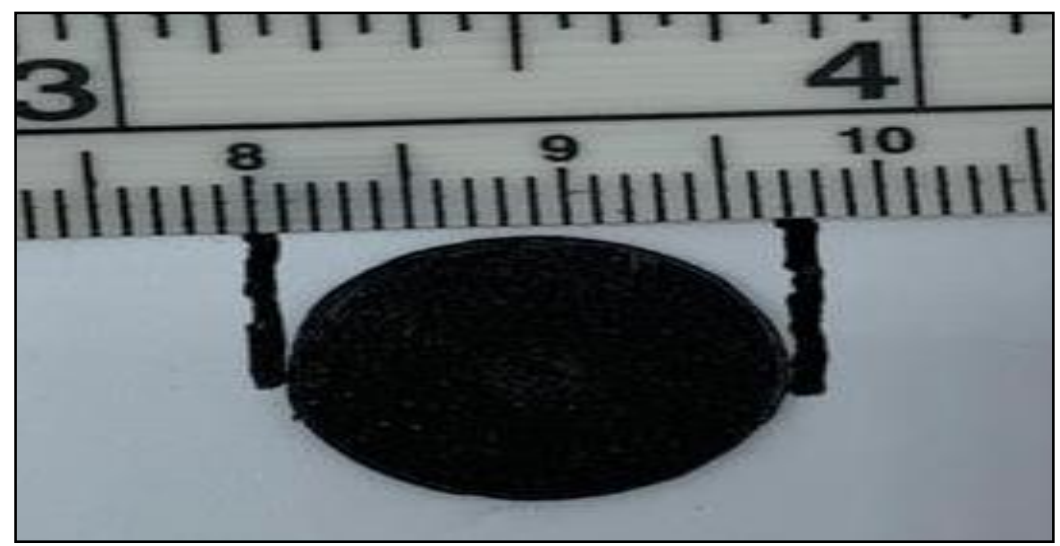

Figure 2: The resulting pellet.

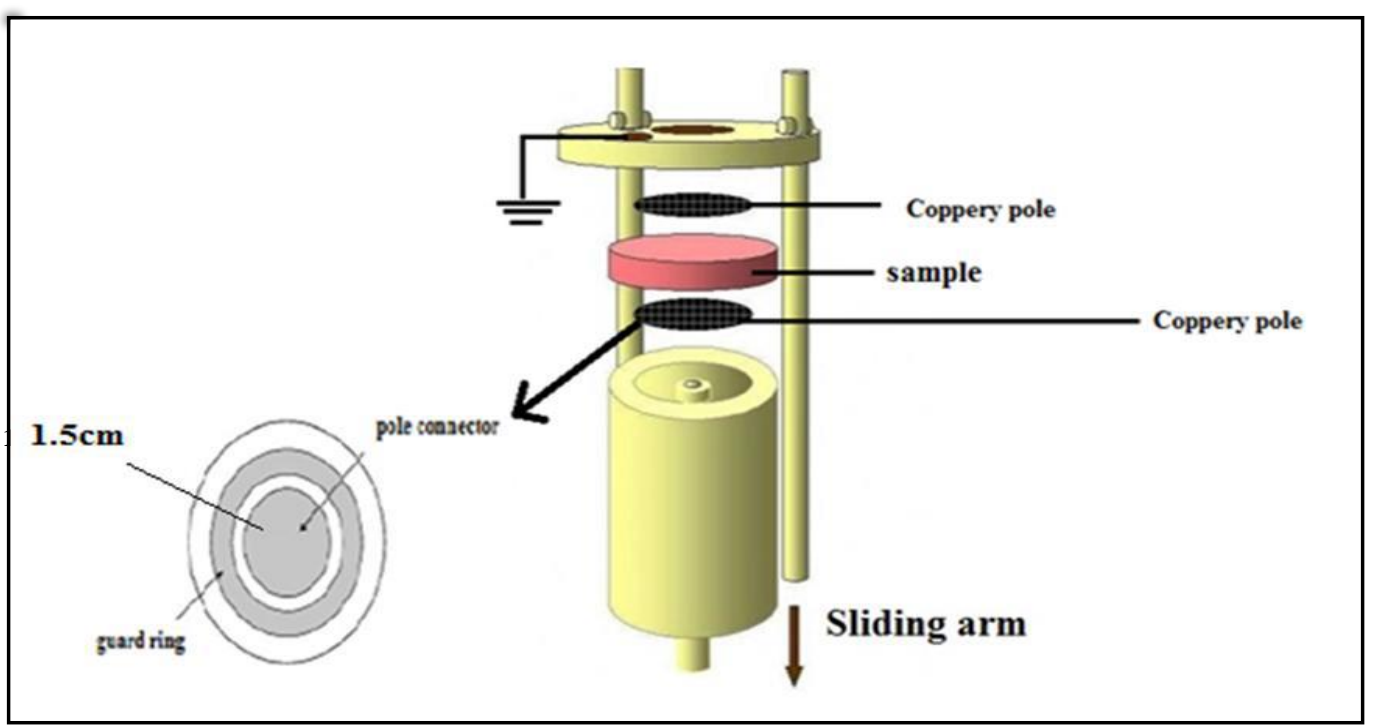

Figure 3: Schematic diagram of used electrode.

\section{Results and discussion}

\subsection{Optical Properties}

The optical properties of PPy and PPy/GN were recorded by UV-visible spectra, in the wavelength range of (200-800) $\mathrm{nm}$. The absorption versus wave length and optical energy gap have been evaluated.

\subsubsection{Absorption of UV-Vis. spectroscopy}

The optical absorption spectrum, obtained by UV-spectroscopy, of PPy and PPy/GN are shown in Figure 4(a-b). The absorption spectra have two peaks (maximum absorbance). One, that can be seen at wavelength $300 \mathrm{~nm}$, belongs to $\pi \rightarrow \pi^{*}$ and the other at $580 \mathrm{~nm}$ is due to $n \rightarrow \pi^{*}$. It was observed that the maximum absorption at the UV region was shifted towards lower wavelengths with increasing the GN concentration, it was at $280 \mathrm{~nm}$ and $590 \mathrm{~nm}$, respectively.

\subsubsection{Optical Energy Gap}

Tauc put the empirical equation of optical energy gap and energy of incident photon which is [9]

$$
\text { ahv } v={ }^{"} A^{n}\left(h v-E_{g}\right)^{r}
$$



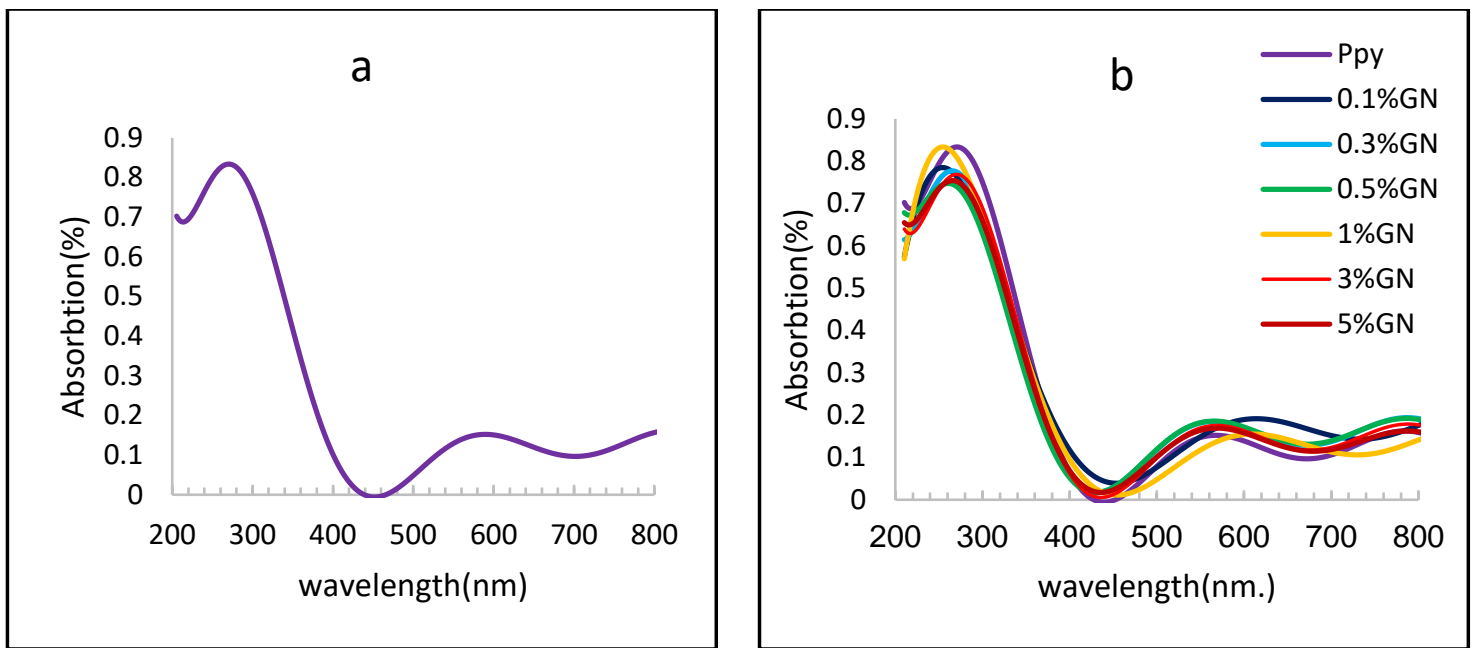

Figure 4: The absorption spectra of (a)pure PPy, (b)PPy/GN nanocomposites.

where $\mathrm{A}$ is a constant, ho energy of incident photon, which can be calculated using the equation $h v=1240 / \lambda(\mathrm{nm})$. And, depending on the type of the electronic transition, $\mathrm{r}$ is the optical transition order. If the extremities of V.B. and C.B. are at the same location in $\mathrm{k}$ - space, the transition is termed direct, but if the transition is only achievable with phonon aided $(\mathrm{k} \neq 0)$, it is called indirect. As a result, the allowed direct, allowed indirect, banned direct, and forbidden indirect transitions have values of $1 / 2,2,3 / 2$, and 3 , respectively [10].
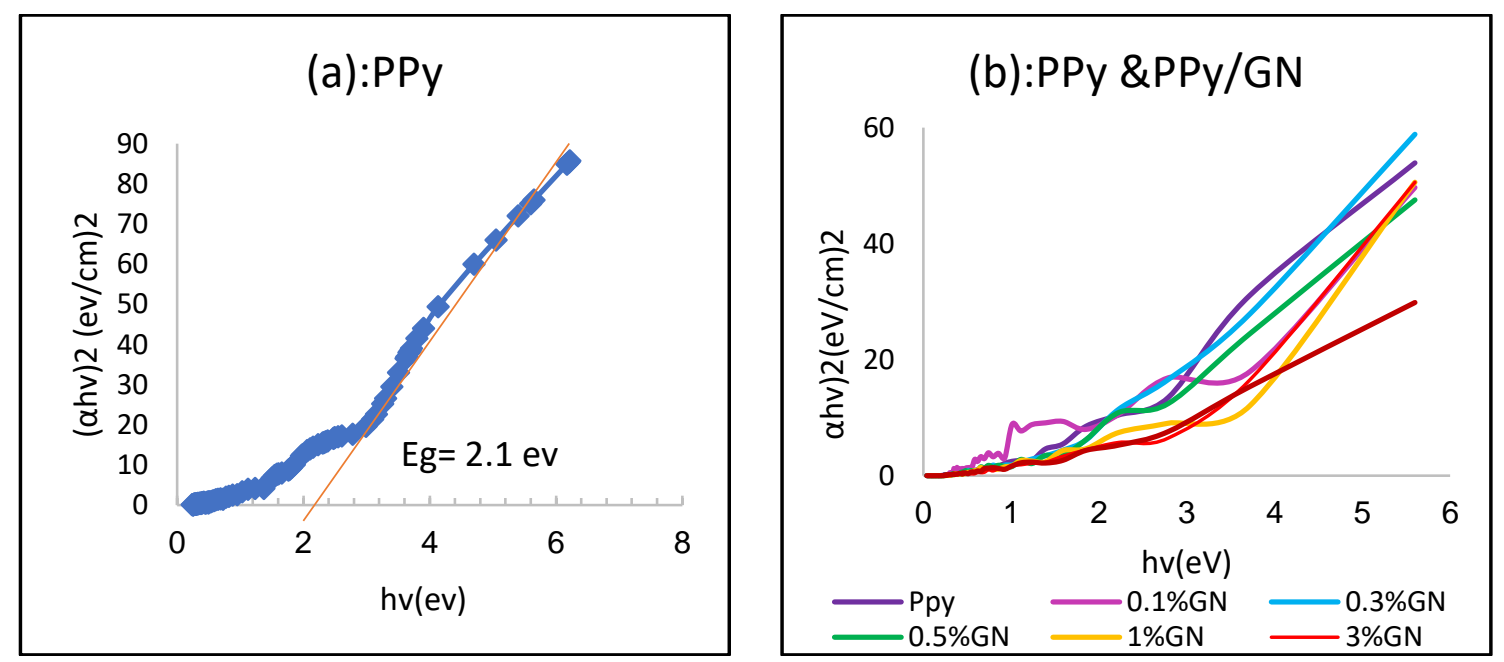

Figure 5: Evaluation of $E_{\text {opt }}$ from optical absorption spectra of: (a) the PPy and (b) the PPy and PPy/GN.

The plots of $(\alpha h v)^{2}$ versus photon energy ho were used to evaluate the optical energy gaps. A typical plot for pure PPy and PPy/GN are shown in Figure (5-a), (5-b), respectively. It can be seen, all samples exhibit a direct optical energy gap, as seen in this diagram. The energy reduced as the concentration \% of GN increased from (2.1-1.3) eV, indicating that the reduction in the optical band gap is probably due to the modification of the polymer structure.

\subsection{A.C. Conductivity}

The A.C. conductivity for conducting polymer changes with frequency according to the following formula [11]:

$$
\sigma_{\mathrm{AC}}(\omega)=\hat{A} \omega^{s}
$$


where: $\grave{A}$ is a constant parameter $\omega=$ angular frequency $=2 \pi \mathrm{f}$ ( $\mathrm{f}$ is the linear frequency), and (s) is the frequency exponent. The conductivity $(\sigma)$, dissipation factor (D) and the resistance $(\mathrm{R})$ were measured with a computerized sensitive impedance analyzer in the frequency range $(10 \mathrm{KHz}-10 \mathrm{MHz})$ at room temperature. Figure (6) shows the frequency dependence of the measured A.C. conductivity for pure PPy and PPy/GN nanocomposites.

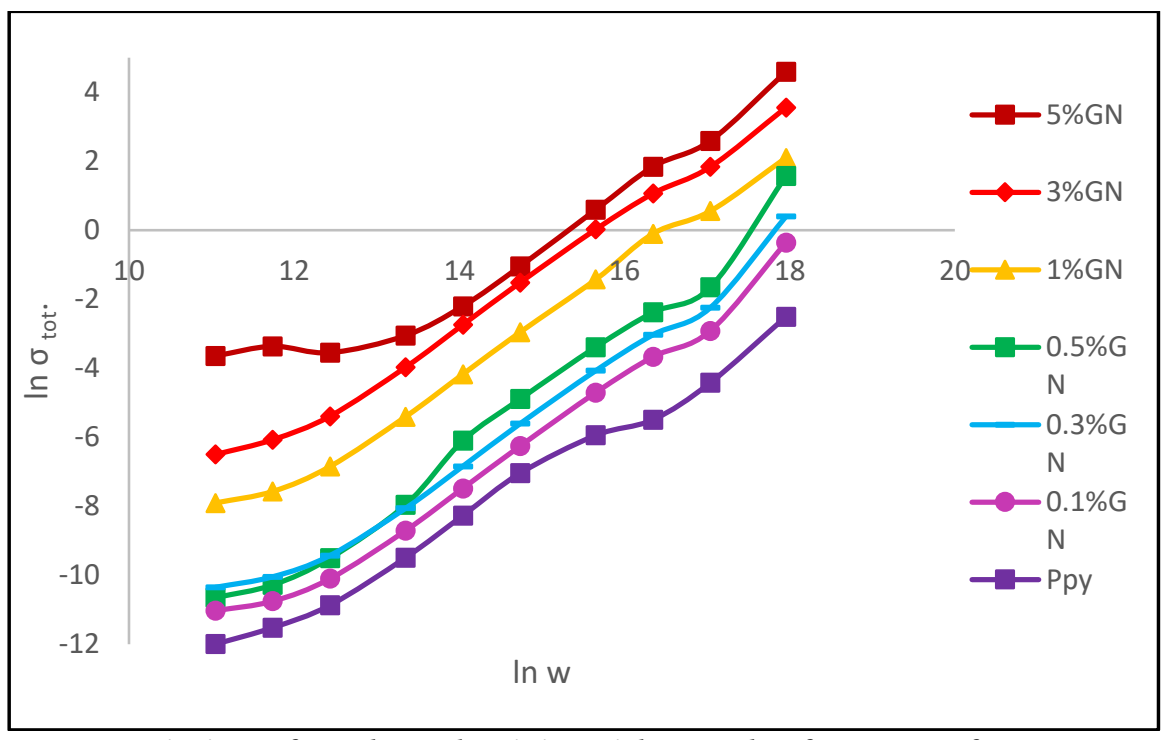

Figure 6: Variation of total conductivity with angular frequency for pure PPy and PPy/GN Nano composites.

The value of A.C conductivity changed from $\left(6.16 \times 10^{-6}-8.1 \times 10^{-2}\right)(\mathrm{S} / \mathrm{cm})$ for pure PPy. The conductivity increased by four orders of magnitude with frequency in the same time the A.C conductivity changed with increasing concentration of GN particles from $\left(1.6 \times 10^{-5}-2.6 \times 10^{-2}\right)(\mathrm{S} / \mathrm{cm})$ when the concentration changed from $(0.1 \% \rightarrow 5 \%)$ at $10 \mathrm{kHz}$, as shown in Figure (7).

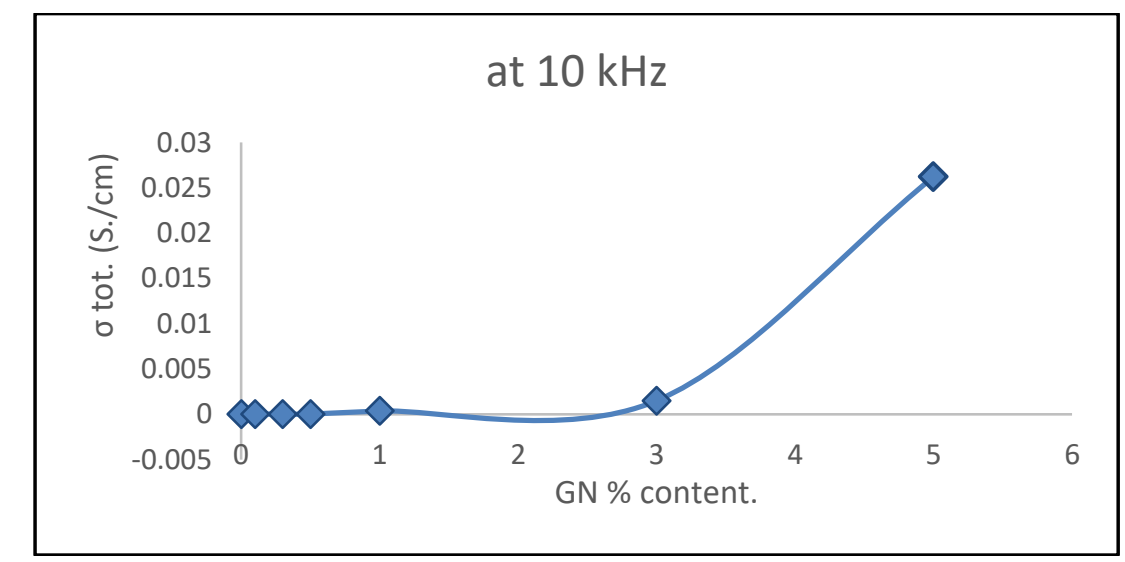

Figure 7: Variation of $\sigma_{\text {tot }}$ as a function of GN concentration at $10 \mathrm{kHz}$.

The conductivity increase is due to the decrease of the hopping barriers as a result of a greater number of densities of state which was introduced by GN in the PPy matrix. The values of the exponent (s) were calculated from the slope of the curves plotted between $\ln \sigma(\boldsymbol{\omega})$ and $\ln (\boldsymbol{\omega})$, as shown in Figure (8) and are listed in Table(1). The exponent (s) was found to be less than unity, (0.65-0.96), and increased with the increase of the concertation of GN, which represented to correlated Hopping mechanism between polymer chain [12]. 


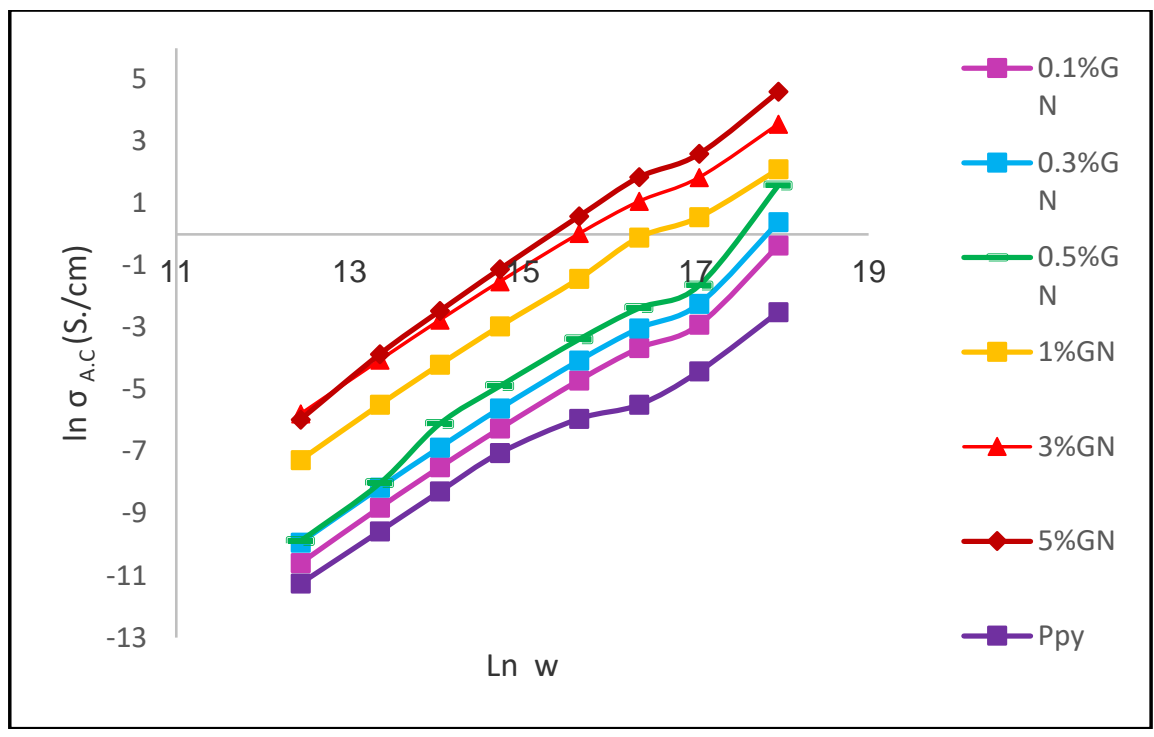

Figure 8: Variation of $\sigma_{A . C}$ with angular frequency for pure PPy and PPy/GN Nanocomposites.

Table 1: The types of samples and its exponent(s) value.

\begin{tabular}{c|c|c|c|c|c|c|c}
\hline Sample & PPy & $0.1 \%$ & $0.3 \%$ & $0.5 \%$ & $1 \%$ & $3 \%$ & $5 \%$ \\
\hline $\mathbf{S}$ & 0.653 & 0.720 & 0.761 & 0.788 & 0.703 & 0.882 & 0.962 \\
\hline
\end{tabular}

\section{Conclusions}

In this section we welcome you to include a summary of the end results of your in this work, polypyrrole polymer was prepared from the oxidation of $(2 \mathrm{ml})$ pyrrole with ( $3 \mathrm{~g})$ of ferric chloride in aqueous medium. Measurements of optical energy gap were done. The minimum optical energy gap value was $(2.1 \mathrm{eV})$ for pure PPy and decreased with increasing the doping level of GN to became $1.3 \mathrm{eV}$ for $5 \%$ wt. All samples have direct energy gap. Measurements of A.C electrical conductivity showed that the conductivity increased by about four orders of magnitude with the increase in frequency and GN concentration.

\section{Acknowledgments}

The authors would like to thanks Department of Physics/College of Science/ in the University of Baghdad for supporting the work in the labs.

\section{Conflict of interest}

Authors declare that they have no conflict of interest.

\section{References}

1. Stejskal J., Trchová M., Bober P., Morávková Z., Kopecký D., Vrňata M., Prokeš J., Varga M., and Watzlová E., Polypyrrole salts and bases: superior conductivity of nanotubes and their stability towards the loss of conductivity by deprotonation. RSC advances, 2016. 6(91): pp. 88382-88391.

2. Saxena R., Sharma K., Saxena N., and Sharma T., Effect of annealing on structural and optical properties of polypyrrole doped with different acids. Polymer composites, 2009. 30(6): pp. 820-826.

3. Gupta S., Price C , and Heintzman E., Conducting polymer nanostructures and nanocomposites with carbon nanotubes: Hierarchical assembly by molecular electrochemistry, growth aspects and property characterization. Journal of nanoscience nanotechnology, 2016. 16(1): pp. 374.391- 
4. Pang A.L., Arsad A., and Ahmadipour M., Synthesis and factor affecting on the conductivity of polypyrrole: a short review. Polymers for Advanced Technologies, 2021. 32(4): pp. 1428-1454.

5. Ganguly S., Bhawal P., Ravindren R., and Das N.C., Polymer nanocomposites for electromagnetic interference shielding: a review. Journal of Nanoscience and Nanotechnology, 2018. 18(11): pp. 7641-7669.

6. Gill N., Gupta V., Tomar M., Sharma A.L., Pandey O., and Singh D.P., Improved electromagnetic shielding behaviour of graphene encapsulated polypyrrole-graphene nanocomposite in X-band. Composites Science Technology, 2020. 192: pp. 108113.

7. Hassan S.M., DC electrical conductivity of prepared pure and doped polyaniline salt. Iraqi Journal of Physics, 2017. 15(33 :(pp. 1-10.

8. Hassan S.M., Optical Properties of Prepared Polyaniline and polymethylmethacrylate blends. International Journal of Application or Innovation in Engineering Management, 2013. 2(9): pp. 232-235.

9. Ajmi A., Karoui K., Khirouni K., and Rhaiem A.B., Optical and dielectric properties of NaCoPO 4 in the three phases $\alpha, \beta$ and $\gamma$. RSC advances, 2019. 9(26): pp. 1477214781.

10. Vandana M., Ashokkumar S., Vijeth H., Yesappa L., and Devendrappa H. Synthesis and characterization of polypyrrole-graphene quantum dots nanocomposites for supercapacitor application. in AIP Conference Proceedings. 2019. AIP Publishing LLC.

11. Abdullah N.J., Essa A.F., and Hasan S.M., Optical and Structural Properties of Prepared Polyaniline â€"Graphene (PANI/GN) Nanocomposite. Iraqi Journal of Science, 2021: pp. 138-146.

12. Hassan S., Ghareeb B., and Jafaar H., AC electrical condutivity for polyaniline prepared in different acidic medium. International journal of basic applied science, 2012. 1: pp. 352-362.

\section{الخواص البصرية و الكهربائية للبولي بيرول المتراكب النانوي البولي بيرول-الكرافين

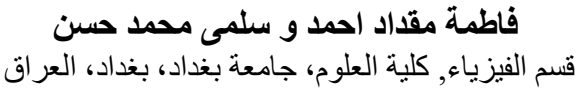

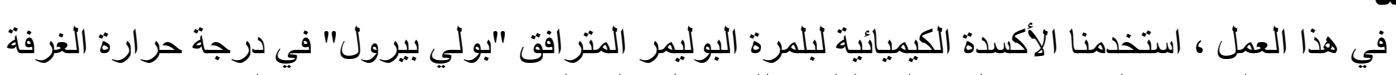

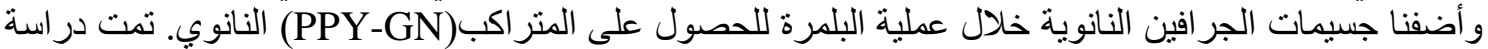

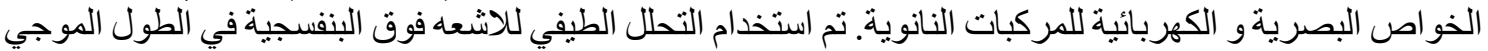

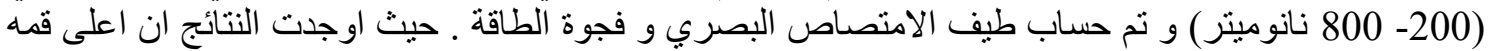

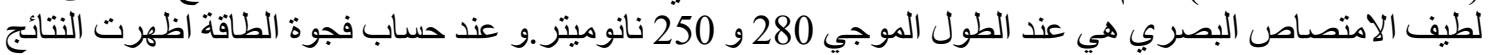

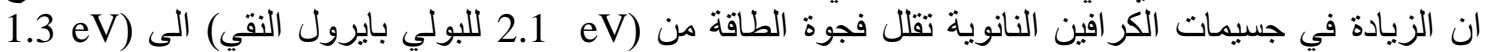

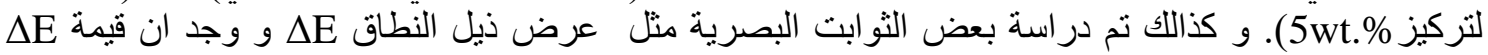

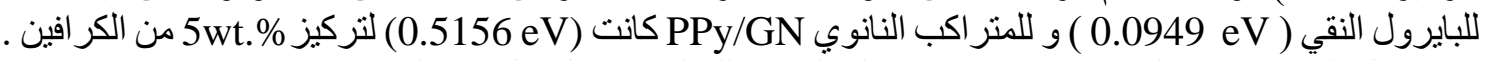

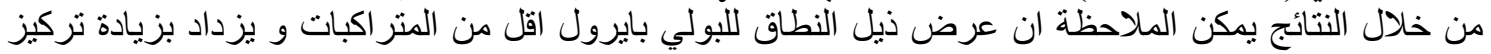

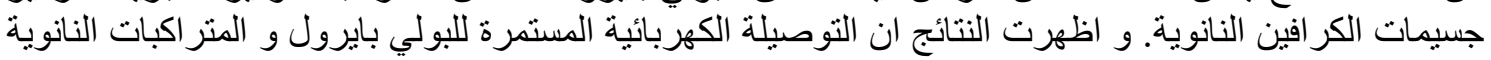

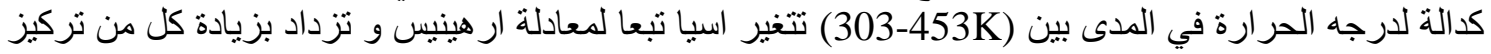

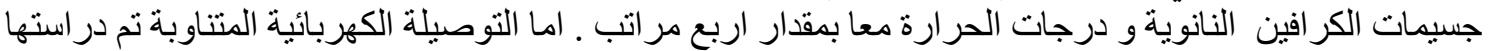
كدالة للتردد ضمن الترددات (1037-106

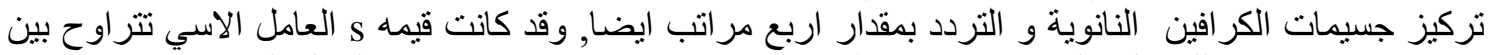

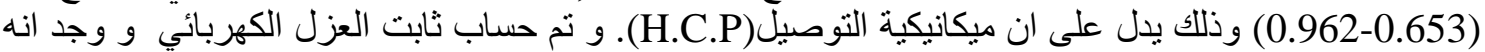
بقل بزيادة التردد. 